caused by a disturbance on the Sun, so that the latcr chapters contain a good deal about the part played by solar emission of particles or radiation.

After an introduction, there is a chapter on the solar optical radiation that affects the ionosphere below $120 \mathrm{~km}$, and then, successively, chapters on the $D$ and $E$ regions of the ionosphere, on the $F$ region, and on the outer ionosphere. The latter includes a review of the motion of charged particles in a magnetic field, prefacing a discussion of the Van Allen belts, those regions whero energetic charged particles are trapped in the geomagnetic field. Next, windlike motions are considered, first of the neutral atmosphere, and then of ionization; the main topics here are tidal oscillations and smaller scale gravity waves (the only purely gas dynamic phenomena treated in the book), and the "dynamo" theory of the diurnal variations of the geomagnetic field. Discussion of the undisturbed atmosphere ends with a chapter jointly on ionospheric noise, that is, audio-frequency radio emissions which produce the delightful medley of sound known as "dawn chorus", and on geomagnetic micropulsations.

Disturbances on the Sun are divided into those emitting ultra-violet and X-radiation, and those emitting charged particles. The former give rise to "sudden ionospheric disturbances", and are dealt with in a single chapter. The latter are thought to be the origin of numerous effects, in particular, polar cap absorption, magnetic storms, and aurorae; and the discussion here occupies a further six chapters. Of these the final one, on storm theory, is an enlightening account of the different suggestions that have been put forward.

The two appendixes give some fifty pages on aspects of electromagnetic wave propagation. They might, perhaps, have been linked a little more closely to the main text, For example, they contain no reference to Faraday rotation, quoted earlier as giving a measure of electron content.

The authors rightly emphasize the incomplete state of knowledge in many of the topics under discussion, and throughout strike a fair balance between rival theories, not a few of which have been contributed by one or other of their company. Their book is to be highly recommended to a wide class of readers.

P. C. Clemmow

\section{SILICATE ANALYSIS AT THE BENCH}

\section{Methods of Silicate Analysis}

By H. Bennett and W. G. Hawley. Second edition. Pp. $\mathrm{xv}+334$. (Published for the British Ceramic Research Association.) (London and New York: Academic Press, 1965.) $63 s$.

For many decades there was little change in the methods of silicate analysis. Standard works such as Hillebrand and Lundell's Applied Inorganic Chemistry, itself based on an earlier text, served a generation of analysts. With the development of instrumental methods and the introduction of new reagents a move was made to introduce simplified methods of silicate analysis. The older, generally applicable, systematic schemes of gravimetric analysis, often lengthy and involved, were replaced by rapid direct determinations using spectro-photo-colorimetric and complexometric methods. Although this process was often accompanied by considerable loss of precision, the fresh impetus given to the subject induced many workers in the field to review methods generally with the result that many of these wore simplified, often with a gain in accuracy.

Although this branch of analysis is now progressive, the once neat pattern of analysis has disappeared, so that these new simplified, but often restricted, methods of analysis exist side by side with the "classical" general methods of analysis. The task for the compiler is therefore not easy and for some time there has been a need for a critical survey of the variety of methods scattcred in reference books and original literature. Bennett and Hawley, in a new and much improved edition of their work, Methods of Silicate Analysis, have gone a long way towards effectively summarizing the present state of silicate analysis, especially in the context of ceramic materials. The title is perhaps slightly misleading, for although materials which are not silicates but are of ceramic interest find a place in this work, certain silicates do not. The work is, in fact, directed primarily at the industrial ceramic analyst rather than the silicate analyst, although it will prove useful to the latter.

The book falls into three sections. Part I is introductory and is followed by a discussion on the basic principles behind the theory and practice of silicate analysis (Part II). The main section (Part III) deals with the analysis of materials; full practical details are given, but general comment and discussion are placed separately in Part II. This makes for easy reading of the practical text. The authors have not hesitated to duplicate material to avoid the necessity for cross-references which would be tiresome and out of place in a work clearly designed to be used at the bench. Chapters in this section of the work are classified on the basis of the material analysed and the general analytical approach adopted. The authors attempt to provide different schemes for different analytical requirements; these depend on the apparatus available, the accuracy required, and the material being analysed.

Materials discussed are those which have some bearing on the ceramic industry such as siliceous and aluminous materials, carbonates, refractories, frits, glazes, etc. Methods described include the generally applicable classical schemes of analysis, accurate direct determinations, and rapid gravimetric, volumetric and spectrophotometric procedures. There are chapters devoted to the analysis of alkalis, the determination of fluorine and to methods connected with the solubility and resistance of materials toward aqueous and acid attack.

The text is thoroughly reliable, for it is abundantly clear that a considerable amount of personal practical experience has gone into this book, and its use should steer an analyst clear of those hidden pitfalls of practical silicate analysis. Although it emphasizes ceramic analysis, the book should prove of value to all silicate analysts provided they are aware of the limitations of certain specialized methods. It is thoroughly to be recommended.

A. D. Wilson

\section{CATIONS AND CATALYSIS}

\section{Friedel-Crafts and Related Reactions}

Edited by George A. Olah. Vol. 1: General Aspects. Pp. xxxiv + 1031. 220s. Vol. 2: Alkylation and Related Reactions. Part 1: Pp. xxix + 1-658. Part 2: Pp. xxv + 659-1362. 378s. the two parts. Vol. 3: Acylation and Related Reactions. Part 1: Pp. xxvi +1-910. Part 2: Pp. xxii +911-1606. 450s. the two parts. Vol. 4: Miscellaneous Reactions; Cumulative Indexes. Pp. xvii +1191. 340s. (New York and London: Interscience Publishers, a Division of John Wiley and Sons, Inc., 1963-65.)

ONE of the ways which is in use at present to render down to digestible proportions the vast mass of chemical information is the publication of multi-author books on a wide theme. Each specialist then selects and presents his own considerations of what he considers to be significant. This procedure, if it is to be suecessful, requires a firm and wise editor who must be able to obtain co-operation from his authors and who must possess the vision which will ensure that the theme is well served.

Dr. Georgo Olah has presided over the production of a magnificent work on, essentially, catalysed cationic reactions. Friedel-Crafts and Related Reactions is the com. bined effort of fifty contributors from nine different 